\title{
Performance Improvement of Wireless Mesh Networks by Using a Combination of Channel-Bonding and Multi-Channel Techniques
}

\author{
Liang XU $^{\dagger \text { a) }}$, Student Member, Koji YAMAMOTO ${ }^{\dagger}$, Hidekazu MURATA ${ }^{\dagger}$, Members, \\ and Susumu YOSHIDA ${ }^{\dagger}$, Fellow
}

\begin{abstract}
SUMMARY In the present paper, the use of a combination of channelbonding and multi-channel techniques is proposed to improve the performance of wireless mesh networks (WMNs). It is necessary to increase the network throughput by broadening the bandwidth, and two approaches to effectively utilize the broadened bandwidth can be considered. One is the multi-channel technique, in which multiple separate frequency channels are used simultaneously for information transmission. The other is the channel-bonding technique used in IEEE 802.11n, which joins multiple frequency channels into a single broader channel. The former can reduce the channel traffic to mitigate the effect of packet collision, while the latter can increase the transmission rate. In the present paper, these two approaches are compared and their respective advantages are clarified in terms of the network throughput and delay performance assuming the same total bandwidth and a CSMA protocol. Our numerical and simulation results indicate that under low-traffic conditions, the channel-bonding technique can achieve low delay, while under traffic congestion conditions, the network performance can be improved by using multi-channel technique. Based on this result, the use of a combination of these two techniques is proposed for a WMN, and show that it is better to use a proper channel technique according to the network traffic condition. The findings of the present study also contribute to improving the performance of a multimedia network, which consists of different traffic types of applications.

key words: IEEE 802.11n, channel-bonding, multi-channel, CSMA/CA, wireless mesh network
\end{abstract}

\section{Introduction}

In recent years, wireless mesh networks (WMNs) have attracted a lot of attention. In this type of network, a mobile station communicates with the nearest AP, which relays the data packets to the mesh portal point (MPP) connected to the Internet. Since all of the links in a WMN are wireless, the network deployment cost can be simply reduced, and the network flexibility such as network coverage and AP placement can be improved. However, since all links are wireless, there may be a lot of interference and packet collisions, which may degrade the network performance seriously. Therefore, it is important to improve the performance of the WMNs by reducing interference and packet collisions. In addition, there may be different multimedia applications, such as text data, Voice over IP (VoIP) data, and video streaming data, generated in the WMN. In this case, different multimedia applications may have different Quality of Service (QoS) requirements. For example, for

\footnotetext{
Manuscript received December 25, 2007.

Manuscript revised April 28, 2008.

${ }^{\dagger}$ The authors are with the Graduate School of Informatics, Kyoto University, Kyoto-shi, 606-8501 Japan.

a)E-mail: xuliang@ @anase.kuee.kyoto-u.ac.jp DOI: 10.1093/ietcom/e91-b.10.3103
}

voice traffic, an end-to-end delay of over $150 \mathrm{~ms}$ is unacceptable to users and the delay between packets should be under $20 \mathrm{~ms}$ for uninterrupted and smooth audio [1]. Although for Video-on-Demand (VoD) streaming, end-to-end delay is not so important compared to live streaming [2], the minimum throughput should be guaranteed. Therefore, it is also necessary to choose the appropriate method of performance improvement according to different multimedia traffic.

In wireless networks, it has been shown that the network performance can be greatly improved by using multiple channels [3], [4]. Unlike a single-channel transmission, a multi-channel transmission allows simultaneous transmissions as long as they use different channels. At the same time, by using the multi-channel technique, the probability of packet collision can be reduced because of the traffic mitigation in each channel. Therefore, it is considered that the multi-channel technique can improve the network performance, and a number of MAC protocols have been proposed for multi-channel transmission systems [5]-[9]. For example, the MAC protocol in [5] brings the concepts of power control and multi-channel technique together to improve the performance of a mobile ad-hoc network (MANET). However, the overhead of these multi-channel MAC protocols is slightly larger than single-channel transmission, which might decrease the transmission efficiency.

Meanwhile, it is well known that increasing the packet transmission rate can also improve the throughput and delay performance, and it can be simply realized by broadening the bandwidth. Therefore, a channel-bonding technique in which multiple frequency channels are joined into a single broader channel is considered. In the IEEE 802.11n standard [10], two separate adjacent channels are bonded into one broader channel to improve the transmission rate.

Comparing these two techniques, we notice that the multi-channel technique aims to reduce packet collisions by using multiple channels, while the channel-bonding technique focuses on realizing a high-data-rate packet transmission by using a broadened channel. Since either of these two techniques has its own advantage, our interest lies in how each technique should be used properly to achieve the best network performance.

Therefore, in the present paper, we first compare the performance of these two techniques in a CSMA network. We present a simple theoretical analysis on the multichannel and channel-bonding CSMA using Kleinrock's the- 
ory [11]. However, at present, an improved MAC protocol CSMA/CA that contributes to Collision Avoidance (CA) is widely used in wireless systems, which is difficult to analyze theoretically. This protocol uses random backoff time and an RTS/CTS exchange mechanism for distributed communication, which might lead to a different conclusion from the theoretical conclusion. Therefore, it is necessary to use computer simulations in a CSMA/CA network to confirm our theoretical conclusion. After that, based on the results, we propose the use of a combination of channel-bonding and multi-channel techniques in a WMN and show the performance improvement by selecting a proper channel technique according to the network congestion mode.

The remainder of the present paper is organized as follows. Section 2 conducts a theoretical analysis of channel-bonding and multi-channel non-persistent CSMA. In Sect. 3, we use computer simulations to evaluate the performance of channel-bonding and multi-channel techniques in a CSMA/CA wireless network. In Sect. 4, we describe the effects of the proposed scheme on the performance improvement using computer simulations and present a discussion of the contribution for multimedia networks. Finally, Sect. 5 concludes our study.

\section{Theoretical Analysis of Multi-Channel and Channel- Bonding CSMA}

\subsection{Analysis Assumptions}

We assume that the transmission range of each node is large enough to communicate with all of the other nodes. For ease of theoretical analysis, in the present paper, each node uses non-persistent CSMA as the MAC protocol, and we will consider the CSMA/CA case in the next section. A constant propagation delay is assumed between each node, and a packet collision occurs if more than one packet is transmitted in the propagation delay period. The network traffic follows a Poisson distribution, and the mean packet arrival rate is $G$ (packets/s). Without loss of generality, we assume the packet transmission time is 1 while using a single channel, according to [11]. The propagation delay, ACKnowledgment (ACK) transmission time, and average retransmission interval are denoted by $a, \alpha$, and $\delta$, respectively.

Furthermore, the total bandwidth used in the channelbonding and multi-channel systems are $n$ times as large as in the single-channel system, as shown in Fig. 1. While using the channel-bonding technique, the $n$ times enlarged bandwidth is used as one channel regardless of the guard band. While using the multi-channel technique, we assume that each node can only use one channel for communication, and there are a total of $n$ single channels that can be used simultaneously. In addition, it is considered that

- the total traffic that occurs in these CSMA networks is the same,

- the data packet size is fixed,

- in the multi-channel CSMA network, the traffic of each channel is the same, and

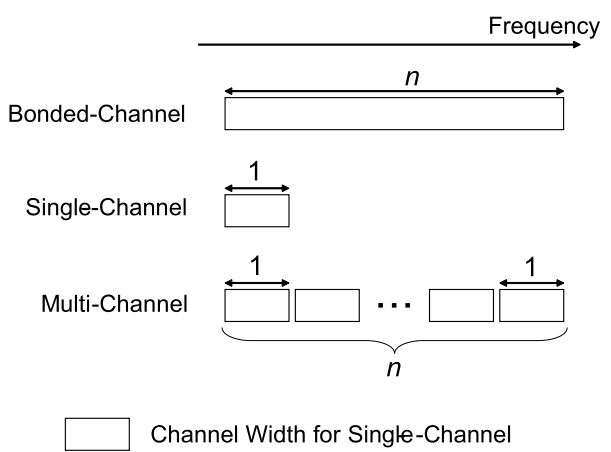

Fig. 1 Bandwidth for channel-bonding and multi-channel transmission.

- in the multi-channel CSMA network, the channel for transmission is set beforehand.

\subsection{Single-Channel CSMA}

According to [11], the average channel utilization (ratio of the data transmission time to the entire time) is given by

$$
E_{\mathrm{s}}=\frac{G \mathrm{e}^{-a G}}{G(1+2 a)+\mathrm{e}^{-a G}} .
$$

Here, the subscript "s" denotes single channel. Since the transmission rate for the single channel is 1 , the throughput is given by

$$
S_{\mathrm{s}}=E_{\mathrm{s}}=\frac{G \mathrm{e}^{-a G}}{G(1+2 a)+\mathrm{e}^{-a G}} .
$$

While analyzing the delay performance, let $R_{\mathrm{S}}$ denote the average retransmission delay between two consecutive transmissions of a given packet. $R_{\mathrm{S}}$ consists of the round-trip propagation delay, the packet transmission time, the ACK transmission time, and the average retransmission interval. Therefore,

$$
R_{\mathrm{S}}=(1+a)+(\alpha+a)+\delta=1+2 a+\alpha+\delta .
$$

Since $\left(G / S_{\mathrm{s}}-1\right)$ is the average number of required retransmissions, the average delay is given as follows:

$$
\begin{aligned}
D_{\mathrm{S}} & =\left(\frac{G}{S_{\mathrm{S}}}-1\right) R_{\mathrm{S}}+1+a \\
& =\frac{G(1+2 a)}{\mathrm{e}^{-a G}}(1+2 a+\alpha+\delta)+1+a .
\end{aligned}
$$

\subsection{Channel-Bonding CSMA}

Compared to the single-channel CSMA, since the channelbonding CSMA uses $n$ times bandwidth, one packet transmission time would be reduced to $1 / n$. Therefore, substituting packet transmission time $1 / n$ into (1), the average channel utilization is given by

$$
E_{\mathrm{b}}=\frac{1 / n \cdot G \mathrm{e}^{-a G}}{G(1 / n+2 a)+\mathrm{e}^{-a G}}=\frac{G \mathrm{e}^{-a G}}{G(1+2 a n)+n \mathrm{e}^{-a G}} .
$$


Here, the subscript "b" denotes channel-bonding. Since the transmission rate for channel-bonding is $n$, the throughput is given by

$$
S_{\mathrm{b}}=n \cdot E_{\mathrm{b}}=\frac{n G \mathrm{e}^{-a G}}{G(1+2 a n)+n \mathrm{e}^{-a G}} .
$$

Therefore, the average delay is given by

$$
\begin{aligned}
D_{\mathrm{b}} & =\left(\frac{G}{S_{\mathrm{b}}}-1\right) R_{\mathrm{b}}+\frac{1}{n}+a \\
& =\frac{G(1+2 a n)}{n \mathrm{e}^{-a G}}\left(\frac{1}{n}+2 a+\frac{\alpha}{n}+\delta\right)+\frac{1}{n}+a .
\end{aligned}
$$

\subsection{Multi-Channel CSMA}

Compared to the single-channel CSMA, since the multichannel CSMA uses $n$ single channels for packet transmission, the traffic in one single channel would be reduced to $G / n$. Therefore, substituting traffic $G / n$ into (1), the average channel utilization is given by

$$
\begin{aligned}
E_{\mathrm{m}} & =\frac{G / n \cdot \mathrm{e}^{-a G / n}}{G / n \cdot(1+2 a)+\mathrm{e}^{-a G / n}} \\
& =\frac{G \mathrm{e}^{-a G / n}}{G(1+2 a)+n \mathrm{e}^{-a G / n}} .
\end{aligned}
$$

Here, the subscript " $m$ " denotes multi-channel. Since there are $n$ channels used for packet transmission, the total throughput is given by

$$
S_{\mathrm{m}}=n \cdot E_{\mathrm{m}}=\frac{n G \mathrm{e}^{-a G / n}}{G(1+2 a)+n \mathrm{e}^{-a G / n}} .
$$

Therefore, the average delay is given by

$$
\begin{aligned}
D_{\mathrm{m}} & =\left(\frac{G}{S_{\mathrm{m}}}-1\right) R_{\mathrm{m}}+1+a \\
& =\frac{G(1+2 a)}{n \mathrm{e}^{-a G / n}}(1+2 a+\alpha+\delta)+1+a .
\end{aligned}
$$

\subsection{Numerical Results}

According to (2), (4), and (6), we show the throughput performance curves with respect to the network traffic of the single-channel technique, the channel-bonding technique, and the multi-channel technique in Fig. 2. Figure 3 shows the delay performance curves according to (2), (4) and (6). Here, we set parameters as $n=2, a=10^{-2}, \alpha=10^{-2}$, $\delta=10^{-1}$ referring to [11].

As shown in these two figures, the channel-bonding technique and the multi-channel technique achieve better throughput and delay performance compared to the singlechannel technique. This is simply because the singlechannel technique uses the half bandwidth of the channelbonding technique and the multi-channel technique.

While comparing the channel-bonding technique and multi-channel technique, when the network traffic is low,

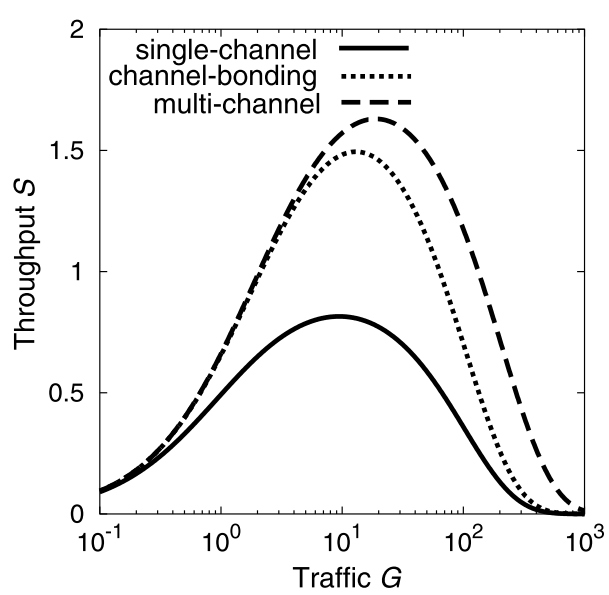

Fig. 2 Throughput performance of channel-bonding and multi-channel CSMA.

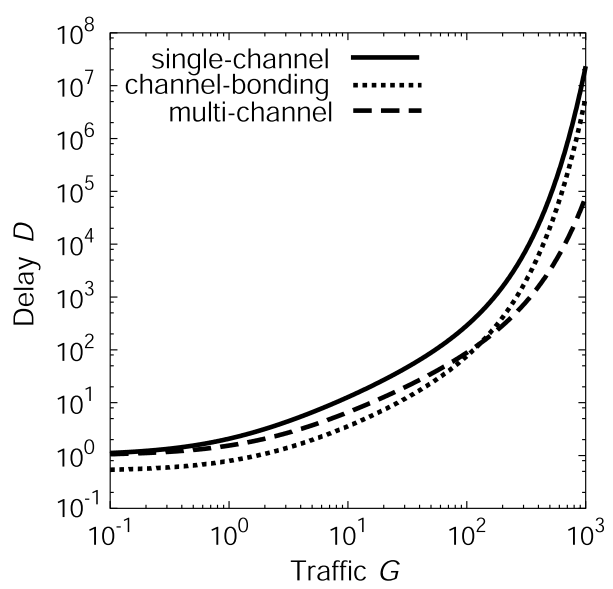

Fig. 3 Delay performance of channel-bonding and multi-channel CSMA.

there is little throughput difference between these techniques. However, the use of channel-bonding can achieve lower delay. In contrast, when the network traffic is high, the throughput performance can be improved by using the multi-channel technique. The reason can be explained as follows:

The delay is considered to be the interval between the time of packet generation and the time of packet reception by the receiver. Therefore, the delay is determined by the packet transmission time and the retransmission time. When the network traffic is low, there are almost no packet retransmissions, so the delay is determined only by the packet transmission time. In this case, channel bonding achieves lower delay because of its short packet transmission time. However, since both the channel-bonding technique and the multi-channel technique use the same total bandwidth, when there are few packet failures, the throughput of both techniques should be the same. For example, if channel-bonding requires one second to send two packets with one channel, then multi-channel transmission also sends two packets in one second with two channels. When the network 
becomes congested, packet collisions increase, which increases the packet retransmission time. While using multichannel transmission, since the traffic of one channel is half that of channel-bonding traffic, the total packet collisions for multi-channel transmission are fewer than for channelbonding transmission. Therefore, the increase in packet retransmission time for multi-channel transmission is less than that for channel-bonding, which causes a smaller difference in delay between these two techniques. In addition, for the same reason, multi-channel transmission achieves higher throughput than channel-bonding transmission.

\section{Simulation Analysis of Multi-Channel and Channel- Bonding CSMA/CA}

We have compared the multi-channel and channel-bonding techniques with non-persistent CSMA. However, an improved protocol CSMA/CA is being used in IEEE 802.11 wireless LAN systems [12]. This protocol can mitigate packet collisions by using a random backoff mechanism, which is not used in the non-persistent CSMA protocol. In addition, while using the multi-channel technique, a multichannel MAC protocol is needed for channel selection, while it is not considered in theoretical analysis. These differences may generate results that differ from the theoretical results. Therefore, it is necessary to show that our conclusion is also appropriate for the CSMA/CA and multichannel MAC protocols.

\subsection{System Model}

The network consists of a certain number of communication flows, and each flow is composed of one sender and one receiver. The transmission range of each node is assumed to be large enough to cover the entire network area. Therefore, each source node can reach its destination directly, disregarding its actual position in the network. The packets of each flow occur according to the Poisson distribution. As a MAC protocol, we use CSMA/CA, which is commonly used in IEEE 802.11 WLAN. The RetryLimit and Lifetime are not considered here, and each packet will be continuously resent until its correct receipt by the receiver.

The multi-channel technique uses two $20-\mathrm{MHz}$ channels for communication and the transmission rate is $54 \mathrm{Mbps}$, while channel-bonding uses one large $40-\mathrm{MHz}$ channel. Therefore, the packet transmission time of channel-bonding transmission would be half that of multichannel transmission due to a doubled bandwidth. While using the multi-channel technique, for ease of analysis, two separate $20-\mathrm{MHz}$ channels without any interchannel interference are considered, and we assume that each sender randomly selects a channel for communication. In addition, a distributed multi-channel MAC protocol, which will be described in greater detail later, is used. Table 1 summarizes all related simulation parameters.
Table 1 Simulation parameters.

\begin{tabular}{c|c}
\hline MAC protocol & CSMA/CA \\
Packet generation & Poisson \\
Data packet rate for single channel & $54 \mathrm{Mbps}$ \\
ACK packet rate for single channel & $24 \mathrm{Mbps}$ \\
RTS/CTS packet rate for single channel & $6 \mathrm{Mbps}$ \\
Data packet size & $1,500 \mathrm{bytes}$ \\
ACK and RTS/CTS packets size & $14 \mathrm{bytes}$ \\
Slot time & $9 \mu \mathrm{s}$ \\
CWmin & 15 \\
CWmax & 1,023 \\
Propagation delay & $0.2 \mu \mathrm{s}$ \\
CCA detect time & $4 \mu \mathrm{s}[14]$ \\
SIFS & $16 \mu \mathrm{s}$ \\
DIFS & $34 \mu \mathrm{s}$ \\
\hline
\end{tabular}

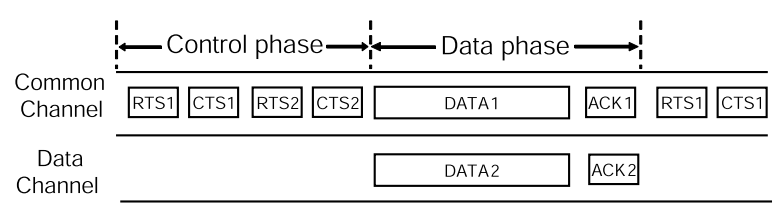

Fig. 4 Multi-channel MAC protocol.

\subsection{Multi-Channel MAC Protocol}

In this protocol, time is divided into an alternating sequence of control and data phases [13]. Here, we assume one channel as the common channel, and this channel can also be used for sending data packets. During the control phase, all nodes are tuned to the common channel, which is the only channel for RTS/CTS exchange, and the channel to be used during the following data phase is decided by RTS/CTS packets.

In the data phase, nodes first tune to the agreed channel and then transfer data packets to the receivers. If packet transmission succeeds, then an ACK packet will be returned from the receiver, or there will be no response from the receiver. After the data phase, all nodes will tune to the control channel again and exchange the RTS/CTS packets to select data channels. In the simulation, such a multi-channel MAC protocol is used.

In this multi-channel MAC protocol, if the packet is successfully received, the control phase time $T_{\mathrm{c}}$ consists of two RTS/CTS time and three SIFS intervals, and thus can be calculated as below:

$$
\begin{aligned}
T_{\mathrm{c}} & =(\mathrm{RTS}+\mathrm{CTS}) \times 2 \times 8 \text { bits } / \text { Rate }_{\mathrm{RTS}}+\mathrm{SIFS} \times 3 \\
& =122 \mu \mathrm{s} .
\end{aligned}
$$

Meanwhile, the data phase time $T_{\mathrm{d}}$ consists one packet, ACK, and SIFS time. Therefore,

$$
\begin{aligned}
T_{\mathrm{d}}= & \text { Packtsize } \times 8 \text { bits } / \text { Rate }_{\text {Data }}+\text { ACKsize } \\
& \times 8 \text { bits } / \text { Rate }_{\text {ACK }}+\text { SIFS } \\
= & 243 \mu \mathrm{s} .
\end{aligned}
$$

Therefore, the overhead of the control phase is $122 /(122+$ 243) $=0.33$. 


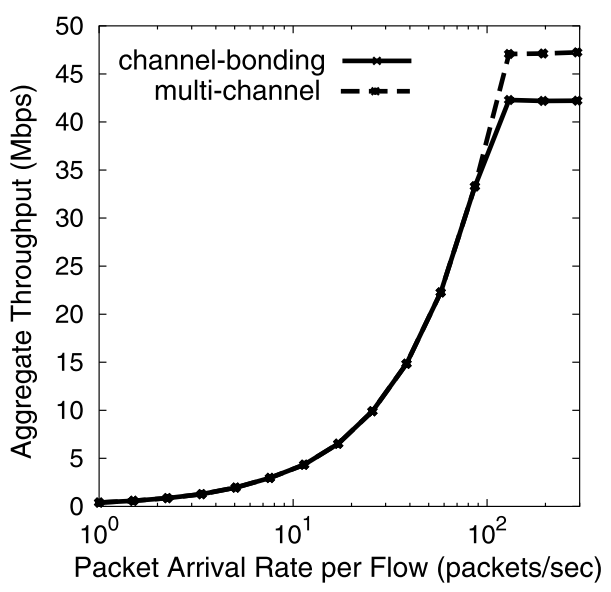

Fig. 5 Throughput versus packet arrival rate.

Compared to this, the overhead for a single channel can be calculated as 0.22 , which is smaller than for multiple channels, because the single channel uses only one RTS/CTS for each data packet. Therefore, the overhead of multiple channels is larger, which may degrade the throughput performance.

Moreover, if the number of channels increases, the overhead of multiple channels will increase, which may cause a more serious throughput decrease for multiple channels. Therefore, in the present paper, in order to obtain the best performance of multiple channels, we assume one common channel and one data channel in the simulation.

\subsection{Simulation Results}

We compare the performance of the channel-bonding and multi-channel techniques with respect to the network traffic. First, we use the packet arrival rate for each flow to vary the traffic and show the results. We then discuss the case in which the number of flows in the network varies.

\subsubsection{Throughput and Delay versus Packet Arrival Rate}

We examine the network throughput and average delay by varying the network traffic. Here, we assume the number of flows is 32 , and use the packet arrival rate per flow to vary the network traffic. Figures 5 and 6 show the aggregate throughput and the average packet delay, respectively. Here, the aggregate throughput represents the total throughput of all flows in the network. The average packet delay is the duration between the time when the packet is generated and the time when the packet is received at the destination. Figure 7 shows the average number of packet retransmission times in the simulation. The average number of packet retransmission times is the total retransmission times divided by the number of successfully transmitted packets.

When the packet arrival rate is low, as can be seen in Fig. 5, there is almost no throughput difference between the channel-bonding and multi-channel techniques. Since the packets are not generated very frequently, the packet

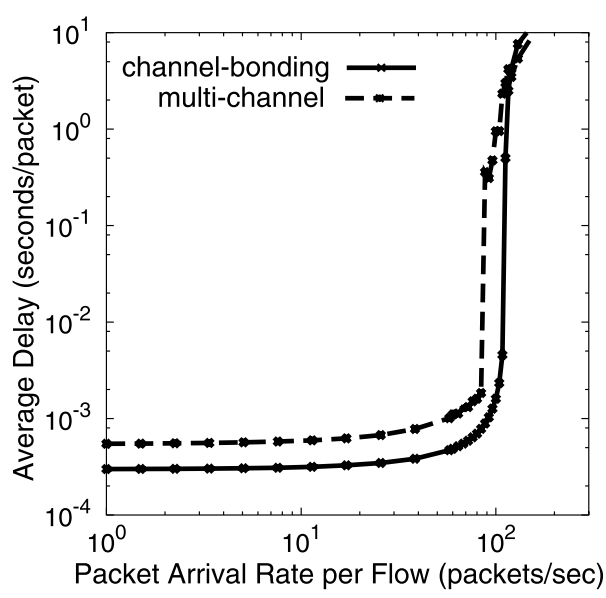

Fig. 6 Delay versus packet arrival rate.

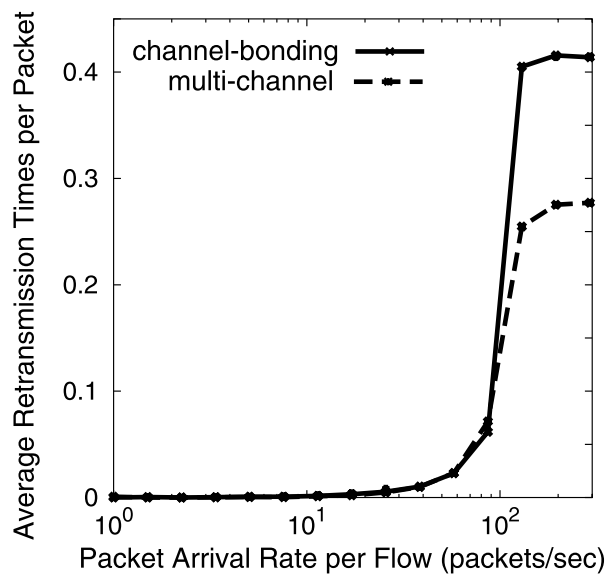

Fig. 7 Average retransmission versus packet arrival rate.

collision can simply be avoided by random backoff in CSMA/CA. Therefore, as can be seen in Fig. 7, there are almost no packet retransmissions. In this case, the throughput is only determined by the packet arrival rate, which is almost the same for each technique. Meanwhile, the delay of the channel-bonding technique is lower, as shown in Fig. 6. Since there are few packet retransmissions, the average packet delay is only determined by the packet transmission time and the overhead. The transmission time of channel-bonding transmission is half that of multi-channel transmission. In addition, in the multi-channel MAC protocol, two RTS/CTS packets should be exchanged for channel determination before transmitting data packets. Therefore, the overhead for each data packet is larger than usual. Therefore, channel-bonding transmission achieves less delay compared to multi-channel transmission.

As the packet arrival rate increases, the number of packet collisions increases, which leads to an increase in the number of packet retransmissions, as shown in Fig. 7. As defined in [7], the average delay is considered to be the interval between the time of packet arrival at the sender, and the time of packet reception by the receiver. Therefore, despite the large number of generated packets, few 


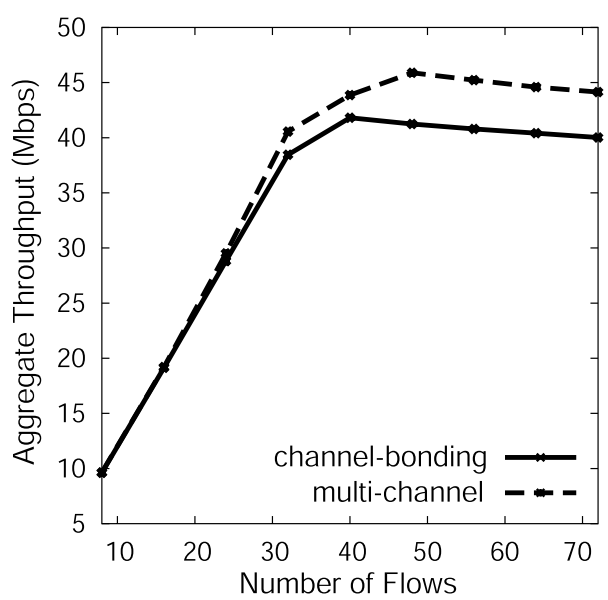

Fig. 8 Throughput versus number of flows.

packets can be transmitted successfully, and most packets are stored in the buffer, which causes a large buffer delay. Therefore, although the average retransmission rate does not increase significantly, the average buffer delay increase is significant for both the multi-channel and channel-bonding techniques. However, the increase in the number of packet collisions of multi-channel transmission is less than that of channel-bonding transmission. This is because it is assumed that each sender randomly selects a channel for communication in advance, so the traffic of each channel is reduced by the use of multiple channels. Therefore, the total probability of packet collisions decreases, which achieves better throughput performance compared to channel-bonding transmission.

\subsubsection{Throughput and Delay versus Number of Flows}

In Sect. 3.3.1, we used the packet arrival rate to vary the traffic. However, the traffic is also related to the number of flows in the network. If the number of flows increases, the number of flows that compete for channel access will increase, which may lead to a more serious collision problem. Therefore, in this section, we vary the number of flows in order to realize variable traffic and analyze the throughput and delay performance. Here, we set the packet arrival rate per flow at 100 packets/s. Figures 8 and 9 show the aggregate throughput and the average packet delay, respectively. Figure 10 shows the average packet retransmission times.

When the number of flows in the network is small, as can be seen in Fig. 10, there are few packet collisions when using CSMA/CA. In this case, the throughput is determined only by the network traffic, which is proportional to the number of flows. Therefore, as shown in Fig. 8, there is almost no throughput difference between channel-bonding transmission and multi-channel transmission. Furthermore, channel-bonding transmission has less delay because of the higher transmission rate and smaller overhead as compared to multi-channel transmission.

As the number of flows increases, packet collisions cannot be avoided completely by using CSMA/CA, and the

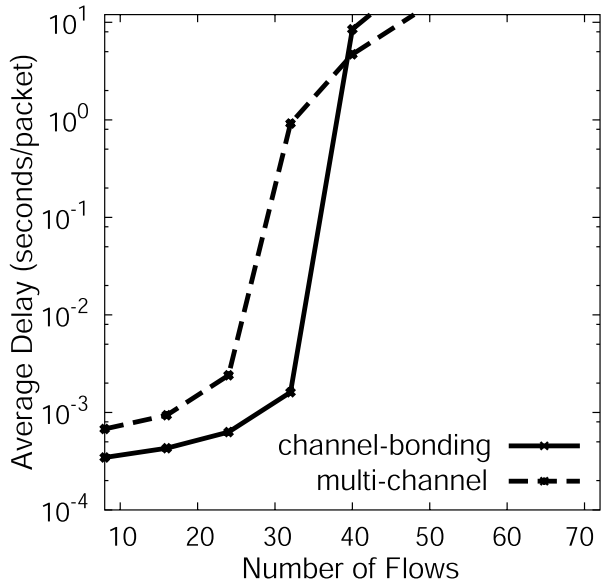

Fig. 9 Delay versus number of flows.

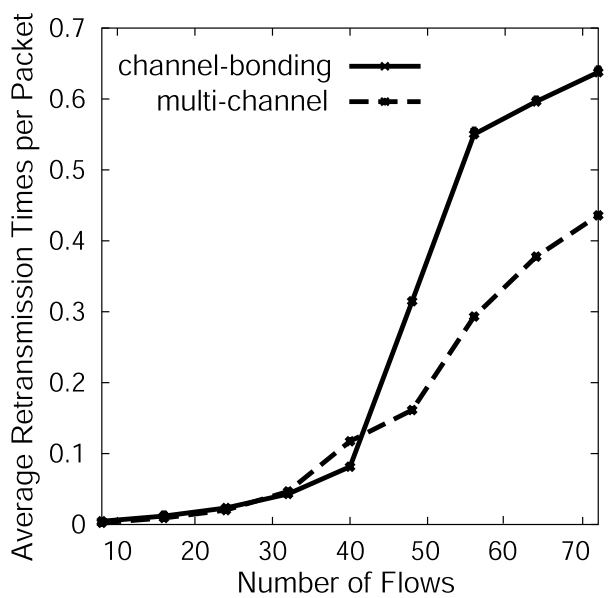

Fig. 10 Average retransmission versus number of flows.

packet retransmission rate increases, as shown in Fig. 10. Therefore, the average delay for both the multi-channel and channel-bonding techniques increases significantly due to the increased buffer delay. However, the increase in the number of packet collisions for multi-channel transmission is less than that for channel-bonding transmission. Therefore, the throughput of multi-channel transmission becomes higher than that of channel-bonding transmission. The average delay is related to the packet transmission and retransmission time. Although the transmission time and overhead of multi-channel transmission are larger than those of channel-bonding transmission, the packet retransmission of multi-channel transmission is less than that of channelbonding transmission. Therefore, the difference in the average delay between channel-bonding transmission and multichannel transmission decreases.

\section{Performance Improvement for WMN Uplink Trans- mission by Combination of Channel-Bonding and Multi-Channel Techniques}

Here, we consider one type of WMN, as shown in Fig. 11, 


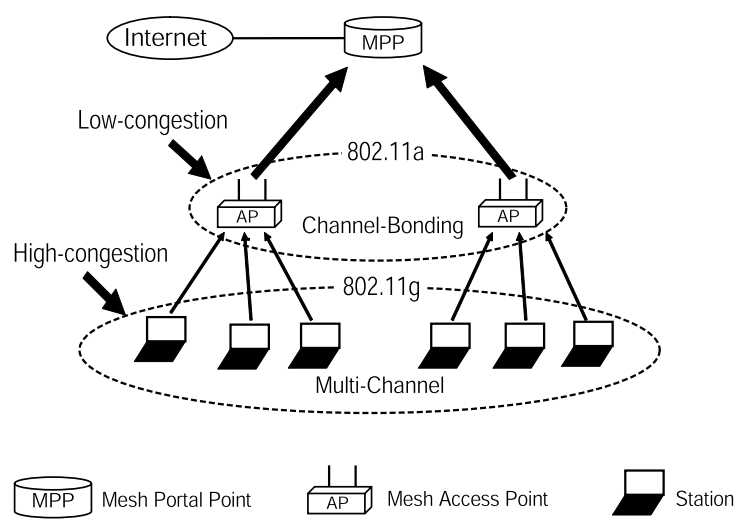

Fig. 11 Proposal for WMN uplink transmission.

which can be commonly seen at office buildings, exhibition halls, and so on. In this mesh network, there are several access points (APs) arranged in fixed locations, and there are a number of mobile stations that want to upload their data packets to the Internet via the APs. The stations use IEEE $802.11 \mathrm{~g}$ at $2.4 \mathrm{GHz}$ to transmit the packets, while the APs use IEEE $802.11 \mathrm{a}$ at $5 \mathrm{GHz}$ to relay the packets to the mesh portal point (MPP), which has wired connection to the Internet. In the present paper, we focus on the performance improvement for WMN uplink transmission, as discussed in [15].

Note that if there are a number of stations that share only one AP, they may compete against each other to obtain the opportunity to transmit data, which may cause network congestion and increase packet collisions. Therefore, we consider it preferable to use the multi-channel technique among stations to reduce the packet collisions. However, since the number of APs is limited, there might be little network congestion among APs. In addition, since an AP collects several packets from stations, it is better to transmit the packets to MPP at a high transmission rate, which can reduce the packet delay. Therefore, we consider the channel-bonding technique to be preferable for AP transmission. Therefore, we propose that multi-channel transmission should be used for station transmission, whereas channel-bonding transmission should be used for AP transmission. In this section, we use computer simulations to show the performance improvement of WMN uplink transmission provided by the proposed technique.

\subsection{System Model}

We assume a WMN having an area of $50 \mathrm{~m} \times 50 \mathrm{~m}$, as shown in Fig. 12. This network consists of one MPP, four APs, and a number of stations. The MPP is located in the center of the network, while the four APs are located at $(15,15),(15,35),(35,15)$, and $(35,35)$, respectively. The stations are randomly located in the network with no mobility. Each station selects its nearest AP to transmit the data packets. The AP that receives the data packets will relay them to the MPP. We assume both stations and APs use CSMA/CA

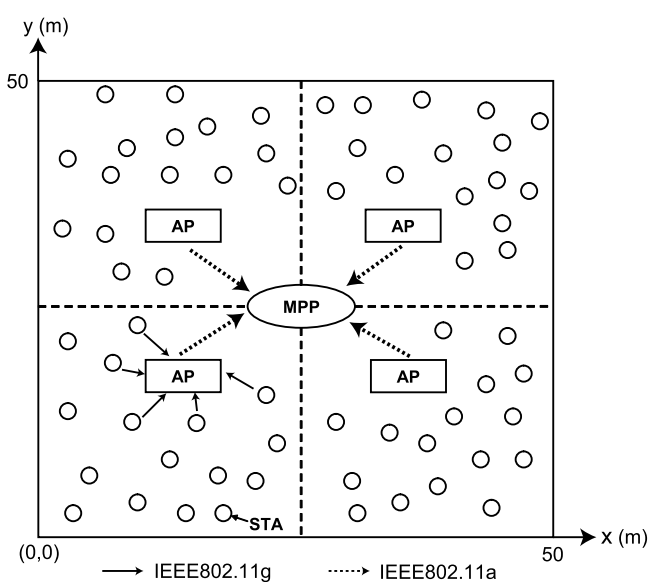

Fig. 12 Simulation network.

as the MAC protocol, and the RTS/CTS exchange mechanism is also considered herein.

It is assumed that IEEE802.11g is used between APs and stations and that IEEE802.11a is used between APs and the MPP. Therefore, an AP can receive and transmit packets simultaneously. Here, we assume both 11a and $11 \mathrm{~g}$ have the same CCA detect time. Multi-channel transmission uses two 20-MHz channels for communication, and the transmission rate is $54 \mathrm{Mbps}$. For ease of analysis, two separate channels without any interchannel interference are considered herein, and we assume that each station or AP selects randomly a channel for communication. In addition, a distributed multi-channel MAC protocol (shown in Fig. 4) is used, and each station randomly selects one channel for data transmission beforehand. While using the channel-bonding technique, each station or AP uses one 40-MHz channel for communication, and the transmission rate is double that of multi-channel transmission. Table 2 summarizes other related simulation parameters. Since stations and APs can use either multi-channel transmission or channel-bonding transmission, there are a total of four types of schemes to transmit packets from the stations to the MPP. Therefore, we will evaluate and compare the throughput and delay performance for each scheme.

\subsection{Simulation Results}

\subsubsection{Throughput and Delay versus Packet Arrival Rate}

We examine the network throughput and average delay by varying the network traffic. Here, we set the number of stations at 100 and use the packet arrival rate at one station to vary the entire network traffic. Figures 13 and 14 show the aggregate throughput and the average packet delay, respectively. In the figures, "multi-bonding" indicates that stations use the multi-channel technique and APs use the channelbonding technique.

When the packet arrival rate is low, as can be seen in Fig. 13, there is almost no throughput difference among the four schemes because when the packets are not gener- 
Table 2 Simulation parameters.

\begin{tabular}{c|c}
\hline Network area & $50 \mathrm{~m} \times 50 \mathrm{~m}$ \\
MAC protocol & CSMA/CA \\
Packet generation & Poisson \\
Transmit power & $17 \mathrm{dBm}$ \\
Carrier sense threshold & $-87 \mathrm{dBm}$ \\
Minimum SINR & $17 \mathrm{~dB}$ \\
Path loss exponent & 3.5 \\
Data packet rate for single channel & $54 \mathrm{Mbps}$ \\
ACK packet rate for single channel & $24 \mathrm{Mbps}$ \\
RTS/CTS packet rate for single channel & $6 \mathrm{Mbps}$ \\
Data packet size & $1,500 \mathrm{bytes}$ \\
ACK and RTS/CTS packets size & $14 \mathrm{bytes}$ \\
Slot time & $9 \mu \mathrm{s}$ \\
CWmin & 15 \\
CWmax & 1,023 \\
CCA detect time & $4 \mu \mathrm{s}[14]$ \\
SIFS & $16 \mu \mathrm{s}$ \\
DIFS & $34 \mu \mathrm{s}$ \\
Simulation time & $100 \mathrm{~s}$ \\
\hline
\end{tabular}

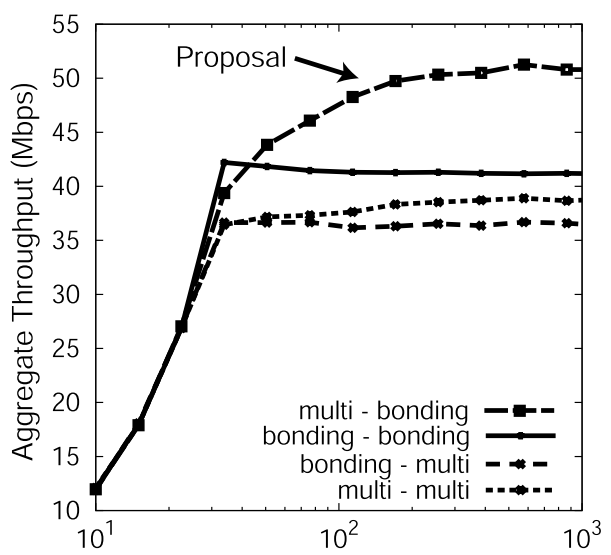

Packet Arrival Rate per Flow (packets/sec)

Fig. 13 Throughput versus packet arrival rate in WMN.

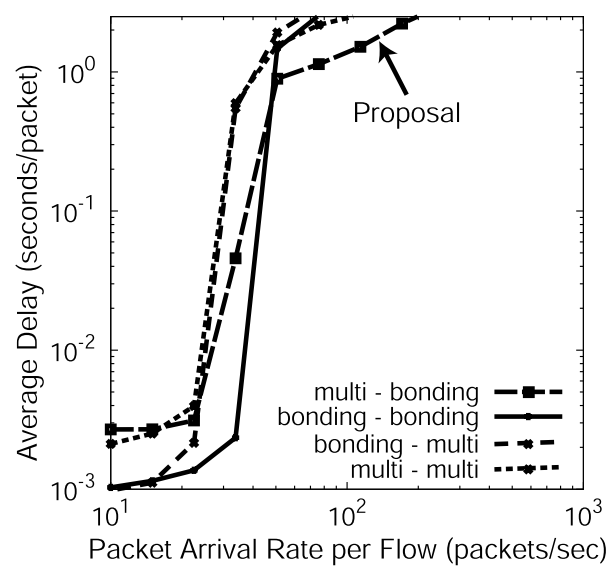

Fig. 14 Delay versus packet arrival rate in WMN.

ated so frequently, there are few packet collisions in the network. Therefore, the use of multi-channel transmission does not significantly affect the improvement of the throughput performance. Meanwhile, as shown in Fig. 14, "bonding- bonding" achieves the lowest delay due to high transmission rate and small overhead. Therefore, in this case, it is better to use the channel-bonding technique throughout the WMN in order to obtain the best delay performance.

When the packet arrival rate is high, as can be seen in Figs. 13 and 14, the proposed "multi-bonding" technique can achieve much higher throughput and lower delay, as compared to the other three schemes. While using multichannel transmission, since the number of stations for one channel is reduced by approximately half, the probability for packet collisions and hidden/exposed terminals will be reduced. Furthermore, interference among stations can also be reduced by using separate different channels. Therefore, multi-channel transmission improves the network throughput and delay performance. However, "multi-multi" does not achieve as high a throughput performance as the proposal technique because there are fewer packet collisions among APs. Therefore, using the multi-channel technique has little effect on improving the AP throughput. In contrast, the multi-channel technique has a slightly larger overhead, as compared to channel-bonding, which may degrade the transmission efficiency. As a result, while both stations and APs use multi-channel transmission, AP throughput might be lower than station throughput, which causes a low end-toend throughput. Therefore, "multi-multi" does not achieve a high throughput performance. Note also that "bondingmulti" achieves the lowest performance for both throughout and delay because it combines the weakness of multichannel transmission and channel-bonding transmission in the WMN.

Note that in Fig. 8, when the number of flows is small, multi-channel and channel-bonding transmission have comparable throughputs, despite the larger overhead of multichannel transmission. Meanwhile, in Fig. 13, the number of APs is 4; however, "multi-bonding" transmission obtains better throughput than "multi-multi" transmission because of the smaller overhead of channel-bonding transmission for AP transmission. The difference between Fig. 8 and Fig. 13 is the packet arrival rate. Since one AP is shared by approximately 25 stations, as shown in Fig. 13, the packet arrival rate of one AP is approximately 2,500 packets/s. However, in Fig. 8, the packet arrival rate for one flow is 100 packets/s. When the flow number is small (such as 4 ) and the packet arrival rate is not high, the channel utilization is empty. Therefore, the throughput of multi-channel transmission is approximately the same as that of channel-bonding transmission, despite the larger overhead of multi-channel transmission, because the throughput is determined only by the packet arrival rate. However, when the packet arrival rate is 2,500 packets/s, the channel utilization will become heavy, while there are few collisions due to the small number of flows. In this case, the effect of multi-channel overhead appears, which leads to a lower throughput compared to channel-bonding transmission. As a result, "multi-bonding" achieves better throughput than "multi-multi." 


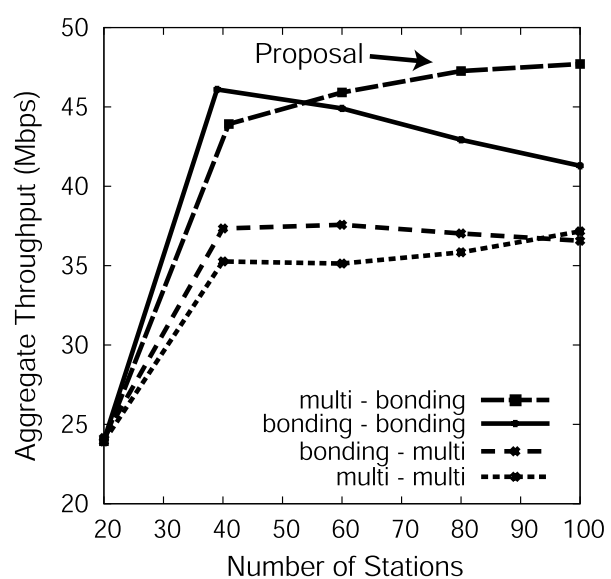

Fig. 15 Throughput versus number of stations in WMN.

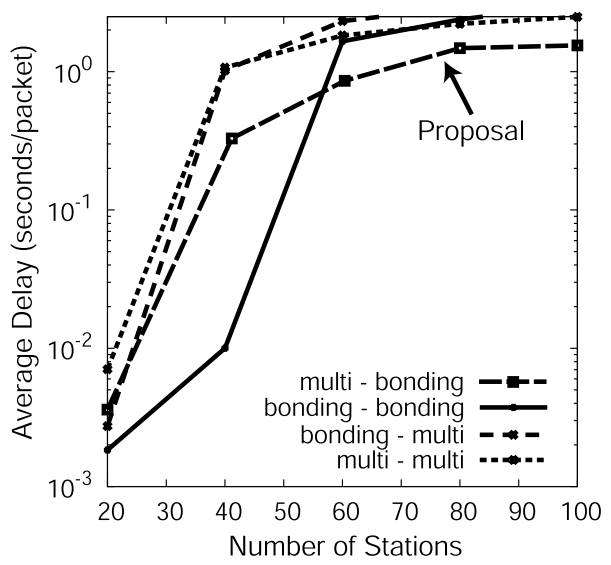

Fig. 16 Delay versus number of stations in WMN.

\subsubsection{Throughput and Delay versus Number of Stations}

We set the packet arrival rate at one station at 100 packets/s, and use the number of stations to vary the network traffic. Figures 15 and 16 show the aggregate throughput and the average packet delay, respectively.

When the number of stations is small, as can be seen in Figs. 15 and 16, "bonding-bonding" achieves the highest throughput and lowest delay because when the number of stations is small, there are few packet collisions due to the CSMA/CA MAC protocol. Therefore, "bonding-bonding," which has a high transmission rate and a small overhead, achieves the highest performance. In this case, the proposed "multi-bonding" technique has a comparable throughput performance to the "bonding-bonding" technique and achieves better network performance, as compared to the other two schemes.

When the number of stations increases, the effect of performance improvement by the proposed technique becomes available. As can be seen in Figs. 15 and 16, "multibonding" achieves the highest throughput and lowest delay among the four schemes. In contrast, the throughput of "bonding-bonding" decreases as the number of stations increases because of the increase in the number of packet collisions. Therefore, the proposed technique is effective to improve the network throughput performance, when there are a number of stations in the network.

\subsection{Contribution for Multimedia Systems}

In multimedia systems, there are different traffic types of applications, such as text data, VoIP data, and video streaming data. These different applications have different requirements for QoS of delay and throughput. For example, VoIP requires a strict constraint of end-to-end delay, but the traffic size is small. Meanwhile, VoD streaming generates large network traffic, and it does not require end-to-end delay stringently, but requires a guaranteed end-to-end throughput. Therefore, according to our results, we can adaptively choose the best scheme according to different types of applications to achieve the best WMN performance. For VoIP packets, the use of "bonding-bonding" can realize the best end-to-end delay performance, while for VoD streaming, "multi-bonding" is the best choice for obtaining the best end-to-end throughput.

\section{Concluding Remarks}

In the present paper, we first used theoretical and simulation analysis to compare the performance between channelbonding and multi-channel techniques. Both numerical and simulation results indicate that when the network traffic is low, the multi-channel technique cannot contribute to throughput improvement, while using the channel-bonding technique can achieve lower delay compared to the multichannel technique. In contrast, when under high-traffic conditions, the multi-channel technique can greatly mitigate the influence of packet collisions, and thus improves the network performance.

Based on these results, we proposed a combination of these two techniques for a WMN to improve the network performance. As the computer simulation results, it is better to use a proper channel technique according to the network traffic condition, and this conclusion also contributes to the improvement of the performance of multimedia network, which consists of different traffic types of applications.

\section{References}

[1] V. Hardman, M.A. Sasse, M. Handley, and A. Watson, "Reliable Audio for use over the Internet," Proc. International Networking Conference (INET'95), pp.171-178, June 1995.

[2] T.T. Do, K.A. Hua, and M.A. Tantaoui, "P2VoD: Providing fault tolerant video-on-demand streaming in peer-to-peer environment," Proc. IEEE ICC, pp.1467-1472, June 2004.

[3] M.A. Marsan and D. Roffinella, "Multichannel local area networks protocols," IEEE J. Sel. Areas Commun., vol.1, no.5, pp.885-897, Nov. 1983.

[4] A. Nasipuri, J. Zhuang, and S.R. Das, "A multichannel CSMA MAC protocol for multihop wireless networks," Proc. IEEE WCNC, pp.1402-1406, Sept. 1999. 
[5] S.L. Wu, Y.C. Tseng, C.Y. Lin, and J.P. Sheu, "A multi-channel MAC protocol with power control for multi-hop mobile ad hoc networks," Comput. J., vol.45, no.1, pp.101-110, 2002.

[6] A. Muir and J.J. Garcia-Luna-Aceves, "A channel access protocol for multihop wireless networks with multiple channels," Proc. IEEE ICC, pp.1617-1621, June 1998.

[7] J. So and N. Vaidya, "Multi-channel MAC for ad hoc networks: Handling multi-channel hidden terminals using a single transceiver," Proc. ACM MobiHoc, pp.222-233, May 2004.

[8] Asis Nasipuri and S.R. Das, "A multichannel CSMA MAC protocol for mobile multihop networks," Proc. IEEE WCNC, pp.1402-1406, Sept. 1999.

[9] R. Garces and J.J. Garcia-Luna-Aceves, "Collision avoidance and resolution multiple access for multichannel wireless networks," Proc. IEEE INFOCOM, pp.595-602, March 2000.

[10] IEEE 802.11n Working Group, "Draft amendment to standard for information technology-telecommunications and information exchange between systems-Local and metropolitan networks-specific requirements - Part 11: Wireless LAN medium access control and physical layer specifications: Enhancements for higher throughput," IEEE P802.11n/D1.0, Jan. 2006.

[11] L. Kleinrock and F.A. Tobagi, "Packet switching in radio channels: Part 1 - Carrier sense multiple-access modes and their throughputdelay characteristics," IEEE Trans. Commun., vol.COM-23, no.12, pp.1400-1416, Dec. 1975.

[12] IEEE 8802.11-1999, "Part 11: Wireless LAN medium access control (MAC) and physical layer (PHY) specifications," 1999.

[13] J. Chen, S. Sheu, and C. Yang, "A new multichannel access protocol for IEEE 802.11 ad hoc wireless LANs," Proc. IEEE PIMRC 2003, vol.3, pp.2291-2296, Sept. 2003.

[14] IEEE Std 802.11a-1999, "Part 11: Wireless LAN medium access control (MAC) and physical layer (PHY) specifications: High speed physical layer in the $5 \mathrm{GHz}$ band," 1999.

[15] M. Cao, V. Raghunathan, and P.R. Kumar, "A tractable algorithm for fair and efficient uplink scheduling of multi-hop wimax mesh networks," Proc. 2nd IEEE Workshop on Wireless Mesh Networks (WiMesh 2006), pp.93-100, Sept. 2006.

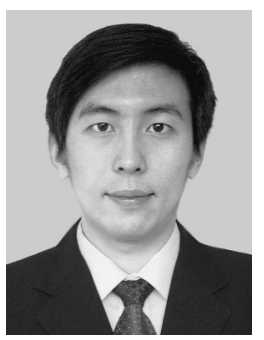

Liang Xu was born in Shenyang, China, in 1981. He received the B.E. degree in electrical and electronic engineering from Kyoto University in 2005, and the M.E. degree from Graduate School of Informatics at Kyoto University in 2007. He is currently studying towards his Ph.D. degree as a Japanese Government Scholarship student at Kyoto University. He received IEICE RCS Active Research Award in 2007.

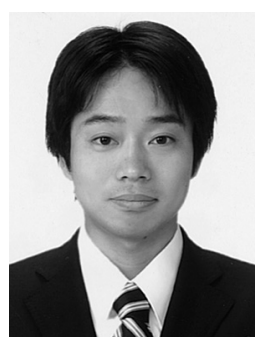

Koji Yamamoto received the B.E. degree in electrical and electronic engineering from Kyoto University in 2002, and the M.E. and Ph.D. degrees in informatics from Kyoto University in 2004 and 2005, respectively. Since 2005, he has been an assistant professor of the Graduate School of Informatics, Kyoto University. Since 2008, he has been a guest researcher at Department of Communication Systems, Royal Institute of Technology $(\mathrm{KTH})$, Sweden. His research interests include cooperative multi-hop radio networks and distributed adaptive controls. He received the PIMRC 2004 Best Student Paper Award in 2004, the Ericsson Young Scientist Award in 2006, and the Young Researcher's Award from the IEICE of Japan in 2008. He is a member of the IEEE.

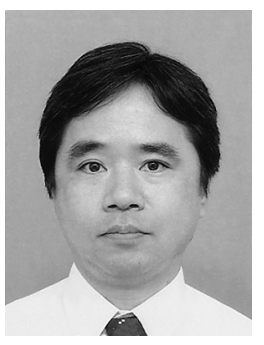

Hidekazu Murata received the B.S., M.S., and Ph.D. degrees in electronic engineering from Kyoto University, Kyoto, Japan, in 1991 , 1993, and 2000, respectively. In 1993, he joined the Faculty of Engineering, Kyoto University. In 2002-2006, he was an associate professor of Tokyo Institute of Technology. Since 2006, he has been an associate professor of Kyoto University. His current research interests include signal processing and its hardware implementation, with particular application to multi-hop cooperative wireless networks. He received the Young Researcher's Award from the IEICE of Japan in 1997, the Ericsson Young Scientist Award in 2000, and the Young Scientists' Prize of the Commendation for Science and Technology by the Minister of Education, Culture, Sports, Science and Technology in 2006. He is a member of the IEEE and SITA.

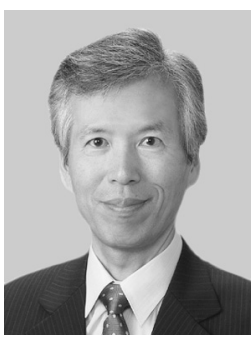

Susumu Yoshida received the B.E., M.E. and $\mathrm{Ph} . \mathrm{D}$. degrees all in electrical engineering from Kyoto University, Kyoto, Japan in 1971, 1973 and 1978, respectively. Since 1973, he has been with the Faculty of Engineering, Kyoto University and currently he is a full professor of the Graduate School of Informatics, Kyoto University. During the last 30 years, he has been mainly engaged in the research of wireless personal communications. His current research interest includes highly spectrally efficient wireless transmission techniques and wireless ad hoc networks. During 19901991, he was a visiting scholar at WINLAB, Rutgers University, U.S.A. and Carleton University in Ottawa. He served as a TPC Chair of IEEE VTC 2000-Spring, Tokyo. He was a guest editor of IEEE J-SAC on Wireless Local Communications published in April and May 1996. He received the IEICE Achievement Award and Ericsson Telecommunication Award in 1993 and 2007, respectively. 